\title{
Quantum corrections in higher spin theory
}

\author{
Arkady Tseytlin ${ }^{1, \star}$ \\ ${ }^{1}$ Blackett Laboratory, Imperial College London, U.K. and Lebedev Institute, Moscow, Russia
}

\begin{abstract}
We review some recent results on computation of quantum corrections in conformal and massless higher spin theory.
\end{abstract}

\section{Introduction}

Below we shall review some recent results of the study of quantum corrections in theories containing infinite number of massless or conformal higher spin states [1-7].

There is a long history of theoretical studies of higher spin theories. Massive HS fields appear in string theory and may become massless in a certain "tensionless" limit (not well defined in flat space). Massless HS theories are known to exist in AdS space and play a natural role in vectorial AdS/CFT duality. Theories dual to free adjoint $U(N)$ CFT's contain both massless tower of states plus infinite set of massive fields in AdS. There is also closely associated theory of conformal higher spins (with nonunitary higher derivative kinetic terms) that admits a consistent in flat-space expansion. Hypothetic theory of massless HS fields in flat space that should have infinite dimensional gauge and global symmetries should have "trivial" S-matrix to comply with analogs of Coleman-Mandula theorem and Weinberg soft theorem.

Theories of infinite number of fields require specific definition at quantum level that should be consistent with their symmetries and provide a prescription of how to sum over all spins. Similar ambiguities in string field theory are fixed by the existence of world-sheet prescription for computation of observables, but in the case of HS theories such a "first-quantized" formulation is not know. To make first steps towards understanding these issues one would like to compute some simple partition functions and scattering amplitudes with higher spin exchanges in massless higher spin (MHS) theory and conformal higher spin (CHS) theory.

In the context of vectorial $\mathrm{AdS}_{d+1} / \mathrm{CFT}_{d}$ duality one starts with a free complex scalar in fundamental representation of $U(N)$, i.e. $\int d^{d} x \partial_{m} \Phi_{i}^{*} \partial_{m} \Phi_{i}(i=1, \ldots, N)$ with a tower of on-shell conserved higher spin currents $J_{s} \sim \Phi_{i}^{*} \partial_{\left(m_{1} \ldots \partial_{m_{s}}\right)} \Phi_{i}+\ldots(s=1,2, \ldots)$ which are $U(N)$ invariant CFT primaries of dimension $\Delta=s+d-2$ which are dual to massless HS fields $\phi_{s}$ field in $\operatorname{AdS}_{d+1} . J_{0}=\Phi_{i}^{*} \Phi_{i}$ with $\Delta=d-2$ is dual to massive scalar with $\Delta(\Delta-d)=m^{2}=-2$. This is the same spectrum as in Vasiliev's massless HS theory in $\mathrm{AdS}_{d+1}$. This MHS theory in AdS "summarizes" correlators of $U(N)$ singlet primaries $J_{s}$ in free CFT: they are reproduced by HS interactions in $\operatorname{AdS}_{d+1}$ with coupling $\sim 1 / N$, i.e. the classical action for Fronsdal fields $\phi_{s}$ in AdS should be

$$
S=N \int d^{d+1} x\left[\sum_{s} \phi_{s}\left(-\nabla^{2}+m_{s}^{2}\right) \phi_{s}+\sum C_{s_{1} s_{2} s_{3}}(\nabla) \phi_{s_{1}} \phi_{s_{2}} \phi_{s_{3}}+\ldots\right] \equiv N \bar{S}
$$

^e-mail: tseytlin@imperial.ac.uk 
Quantum corrections are described by an effective action $\Gamma=N \bar{S}+\Gamma_{1}+N^{-1} \Gamma_{2}+\ldots$. On the CFT side we may consider the generating functional for correlators of currents $J_{s}(\Phi)=\Phi_{i}^{*} \mathcal{J}_{s} \Phi_{i}, \mathcal{J}_{s} \sim \partial^{s}$ introducing source fields $h_{s}$. These will have the interpretation of conformal higher spin (CHS) fields that are gauge fields for the infinite dimensional algebra of symmetries of the free scalar theory. Their linearized gauge transformations $\delta h_{m_{1} \cdots m_{s}}=\partial_{\left(m_{1}\right.} \varepsilon_{\left.m_{2} \cdots m_{s}\right)}+\eta_{\left(m_{1} m_{2}\right.} \alpha_{\left.m_{3} \cdots m_{s}\right)}$ are given by linearized differential and algebraic symmetries that generalize diffeomorphism and Weyl symmetry of the conformal $C^{2}$ gravity. Then the generating functional for connected correlators of currents is found by integrating over free fields $\Phi_{i}$

$$
\Gamma[h]=N \log \operatorname{det}\left(-\partial^{2}+\sum_{s} h_{s} \mathcal{J}_{s}\right)
$$

From the AdS/CFT point of view $\Gamma[h]$ should follow from the massless HS theory in $\mathrm{AdS}_{d+1}$ upon integrating over $\operatorname{AdS}_{d+1}$ fields $\phi_{s}$ with Dirichlet b.c.

$$
e^{-\Gamma[h]}=\int_{\left.\phi_{s}\right|_{\partial \mathrm{AdS}}=h_{S}}\left[d \phi_{s}\right] \exp (-N \bar{S}[\phi])
$$

This matching suggests that all quantum corrections to AdS effective action $\Gamma-N \bar{S}=\Gamma_{1}+N^{-1} \Gamma_{2}+\ldots$ should vanish, i.e. the quantum MHS theory in AdS should be defined so that to satisfy this. Summing up infinite sets of (integrated) correlators suggests also further conjecture: partition function of the CFT on a non-trivial background should match the MHS partition function in (asymptotically) AdS with the corresponding boundary. Then all quantum corrections $\left(N^{0}, N^{-1}, \ldots\right)$ to the AdS partition function should vanish to all orders. In particular, matching at 1-loop order requires $\Gamma_{1}=0$.

This suggests that massless HS theories should have hidden simplicity due to large underlying symmetry (i.e. in a sense are similar to "topological" or "integrable" models). Below we shall discuss some examples of quantum computations in massless and conformal HS theories that demonstrate this.

\section{Free massless HS theory in flat space and in AdS}

One may consider a collection of free massless HS $s=0,1,2,3, \ldots, \infty$ fields with gauge-invariance $\delta \phi_{m_{1} \ldots m_{s}}=\partial_{\left(m_{1}\right.} \epsilon_{\left.m_{2} \ldots m_{s}\right)}$ that are described by the Fronsdal action $S=\int d^{4} x \partial^{n} \phi^{m_{1} \ldots m_{s}} \partial_{n} \phi_{m_{1} \ldots m_{s}}+\ldots$ Like massless vector, graviton, etc. for $s>0$ they have 2 dynamical d.o.f. in 4 d. One then observes a curious fact: the total number of of d.o.f. is zero if each spin enters once and te sum is defined using zeta-function regularization: $1+\sum_{s=1}^{\infty} 2=1+2 \zeta(0)=0$. Equivalent statement is true for free partition function. For each spin $s$ we have

$$
Z_{\mathrm{MHS}, s}=\left[\frac{\operatorname{det} \Delta_{s-1 \perp}}{\operatorname{det} \Delta_{s \perp}}\right]^{1 / 2}=\left[\frac{\left(\operatorname{det} \Delta_{s-1}\right)^{2}}{\operatorname{det} \Delta_{s} \operatorname{det} \Delta_{s-2}}\right]^{1 / 2}=\left(\frac{1}{\sqrt{\operatorname{det}\left(-\partial^{2}\right)}}\right)^{2}
$$

where $\Delta_{s}=-\partial^{2}$ defined on symmetric rank $s$ traceless tensor. Then the total partition function is [3]

$$
Z_{\mathrm{MHS}}=\prod_{s=0}^{\infty} Z_{\mathrm{MHS}, s}=\left[\frac{1}{\operatorname{det} \Delta_{0}}\right]^{1 / 2}\left[\frac{\operatorname{det} \Delta_{0}}{\operatorname{det} \Delta_{1 \perp}}\right]^{1 / 2}\left[\frac{\operatorname{det} \Delta_{1 \perp}}{\operatorname{det} \Delta_{2 \perp}}\right]^{1 / 2}\left[\frac{\operatorname{det} \Delta_{2} \perp}{\operatorname{det} \Delta_{3 \perp}}\right]^{1 / 2} \ldots=1
$$

Here we see cancellation between the physical spin $s$ determinant and the ghost determinant for spin $s+1$ giving the total $Z=1$. This is similar to what happens in a supersymmetric or topological theory. Here this cancellation reflects the large gauge symmetry of the theory. The cancellation of an infinite number of factors is of course a priori ambiguous: it assumes a particular regularization that should be consistent with the underlying symmetry. The formal cancellation of factors we assumed is, in fact, consistent with the above zeta-function prescription for count of d.o.f: $Z_{\mathrm{tot}}=\left(Z_{0}\right)^{v_{\mathrm{tot}}}, v_{\mathrm{tot}}=$ 
$1+\sum_{s=1}^{\infty} 2=0$. This zeta-function prescription is similar to the one used in computation of vacuum energy in bosonic string theory which is consistent with target space symmetries of the theory (the massless vector in $d=26$, etc.). Equivalent to zeta-function is the prescription where one introduces a particular exponential cutoff in the sum over spins and then drops all singular terms. In general dimension $\mathrm{d}$ this reads

$$
v_{\text {tot }}=1+\left.\sum_{s=1}^{\infty} v_{s} e^{-\epsilon\left[s+\frac{1}{2}(\mathrm{~d}-4)\right]}\right|_{\epsilon \rightarrow 0, \text { fin. }}=0
$$

where in $\mathrm{d}=4$ we have $v_{s}=2$.

The fact of triviality of the partition function applies also to the MHS theory in $\mathrm{AdS}_{\mathrm{d}}$ dual to a free scalar CFT in $d$ dimensions $(\mathrm{d}=d+1)$, i.e. $Z=1$ holds in AdS vacuum of Vasiliev theory. The 1-loop partition function is given by the product of the contributions of spin $s$ Fronsdal fields in AdS

$$
\begin{array}{ll}
Z_{\mathrm{MHS}}=\prod_{s=0}^{\infty} Z_{\mathrm{MHS}, s}, & Z_{\mathrm{MHS}, s}=Z_{s, s-1}=\left[\frac{\operatorname{det} \Delta_{s-1 \perp}\left(M_{s-1, s}^{2}\right)}{\operatorname{det} \Delta_{s \perp}\left(M_{s, s-1}^{2}\right)}\right]^{1 / 2}, \\
\Delta_{s}\left(M_{s, k}^{2}\right) \equiv-\nabla_{s}^{2}+M_{s, k}^{2}, & M_{s, k}^{2}=-s+(k-1)(k+\mathrm{d}-2), \quad k=0,1, \ldots ., s-1 .
\end{array}
$$

One may define the determinants using spectral zeta-function so that ( $\Lambda=\mathrm{UV}$ cutoff, $r=\mathrm{AdS}$ radius)

$$
\ln \operatorname{det} \Delta_{s}=-\zeta_{\Delta_{s}}(0) \ln \left(\Lambda^{2} r^{2}\right)-\zeta_{\Delta_{s}}^{\prime}(0)
$$

The natural prescription where one first sums over $s$ for finite argument $z$ of the zeta-function and then takes $z \rightarrow 0$ appears to be consistent with HS symmetries and one finds [3, 8]

$$
\zeta_{\text {tot }}(z)=\sum_{s=0}^{\infty} \zeta_{\Delta_{s}}(z), \quad \zeta_{\text {tot }}(z \rightarrow 0)=0+0 \times z+O\left(z^{2}\right), \quad Z_{\mathrm{MHS}}\left(\operatorname{AdS}_{\mathrm{d}}\right)=1
$$

An equivalent regularization prescription is same as in (6)

$$
\ln Z_{\mathrm{MHS}}\left(\mathrm{AdS}_{\mathrm{d}}\right)=\left.\sum_{s=0}^{\infty} \ln Z_{\mathrm{MHS}, s} e^{-\epsilon\left[s+\frac{1}{2}(\mathrm{~d}-4)\right]}\right|_{\epsilon \rightarrow 0, \text { fin. }}=0
$$

A natural conjecture is that the same triviality applies to all all-loop vacuum partition function: $Z_{\text {MHS }}\left(\operatorname{AdS}_{\mathrm{d}}\right)=1$ : this is required for consistency with the vectorial AdS/CFT duality to free $U(N)$ scalar theory at the boundary.

\section{Conformal higher spin theory}

Conformal higher spins generalize Maxwell $(s=1)$ and Weyl $(s=2)$ theories and describe pure spin $s$ states off shell - they have maximal gauge symmettry consistent with locality. That requires higher-derivative kinetic terms. The $4 \mathrm{~d}$ CHS theory being a consistent local gauge theory with infinite dimensional higher spin symmetry which is closely associated to massless HS theory in $\mathrm{AdS}_{5}$ is of interest as a model regardless the issue of its apparent non-unitarity. Free CHS field in flat $4 \mathrm{~d}$ has the action $S_{s}=\int d^{4} x h_{s} P_{s} \partial^{2 s} h_{s}$ where $P_{s}$ is projector to transverse traceless totally symmetric rank $s$ field. The CHS field $h_{s}$ thus has dimension $2-s$ which is the same as of sources or "shadow" operators for spin $s$ conserved currents $J_{s}(\Phi)$ that have dimension $2+s$. $h_{s}$ also play the role of boundary values for the MHS fields in $\mathrm{AdS}_{d+1}$ dual to $J_{s}$. 
The interacting CHS theory may be defined as "induced" one starting with $I=\int d^{4} x\left[\partial \Phi^{*} \partial \Phi+\right.$ $\left.\sum_{s} \Phi^{*} \mathcal{J}_{s} \Phi h_{s}\right]$ and keeping the local log UV divergent part of the generating functional in (2)

$$
S_{\mathrm{CHS}}=\left.\log \operatorname{det}\left(-\partial^{2}+\sum_{s} \mathcal{J}_{s} h_{s}\right)\right|_{\log \Lambda}
$$

The corresponding partition function in flat $d=4$ space counting effective d.o.f. is given by

$$
\begin{array}{rlrl}
Z_{\mathrm{CHS}, s} & =\left(Z_{0}\right)^{v_{s}}=\left(\operatorname{det} \Delta_{0}\right)^{-v_{s} / 2}, & v_{s}=s(s+1)=2,6, \ldots \\
Z_{\mathrm{CHS}}=\prod_{s=0}^{\infty}\left(Z_{0}\right)^{v_{s}}=\left(Z_{0}\right)^{v_{\mathrm{tot}},} & v_{\mathrm{tot}}=\sum_{s=0}^{\infty} v_{s}
\end{array}
$$

Using again the same regularization

$$
\left.\sum_{s=0}^{\infty} F(s) \rightarrow \sum_{s=0}^{\infty} F(s) e^{-\epsilon\left(s+\frac{d-3}{2}\right)}\right|_{\epsilon \rightarrow 0, \text { fin. }}=0
$$

we find $v_{\text {tot }}=0$ and thus $Z_{\mathrm{CHS}}=1$. This regularization is the same as implied by the relation of MHS theory in $\mathrm{AdS}_{d+1}$ and CHS theory at the boundary. In fact, the 1-loop $Z_{\mathrm{CHS}}\left(S^{4}\right)$ in same regularization is again found to be equal to 1 . This is consistent with the relation to the ratio of MHS partition functions in $\mathrm{AdS}_{5}$ with two choices $(\mathrm{D} / \mathrm{N})$ of boundary conditions [5]

$$
Z_{\mathrm{CHS}, s}\left(S^{4}\right)=\frac{Z_{\mathrm{MHS}, s}^{-}\left(\mathrm{AdS}_{5}\right)}{Z_{\mathrm{MHS}, s}^{+}\left(\mathrm{AdS}_{5}\right)}
$$

Then $Z_{\mathrm{CHS}}\left(S^{4}\right)=1$ is directly related to $Z_{\mathrm{MHS}}^{ \pm}\left(\mathrm{AdS}_{5}\right)_{\mathrm{tot}}=1$. This regularization is consistent with symmetries of the CHS theory: it leads also to the vanishing of conformal anomaly after summation over all spins [7]. For each spin $s$ field one finds

$$
T_{m}^{m}=-\mathrm{a} R^{*} R^{*}+\mathrm{c} C^{2}, \quad \mathrm{a}_{s}=\frac{1}{720} v_{s}^{2}\left(14 v_{s}+3\right), \quad \mathrm{c}_{s}-\mathrm{a}_{s}=\frac{1}{720} v_{s}\left(15 v_{s}^{2}-45 v_{s}+4\right), \quad v_{s}=s(s+1)
$$

and then using the same regularization (15) one gets

$$
\sum_{s=1}^{\infty}\left(\mathrm{c}_{s}-\mathrm{a}_{s}\right)=0, \quad \sum_{s=1}^{\infty} \mathrm{a}_{s}=0
$$

Thus the total conformal anomaly vanishes providing a check of 1-loop quantum consistency of the CHS theory (where Weyl symmetry is one of the gauge symmetries). The summation over infinite set of spins spins using this particular prescription provides a novel mechanism of getting UV finiteness in a bosonic theory (not completely dissimilar to the one in string theory).

\section{Scalar scattering via conformal higher spin exchange}

The issue of regularization of the sum over spins appears again in the context of S-matrix. One example is the scattering of external conformal scalars interacting through exchange of higher spin particles (here conformal, compared to massive tower in string theory). One may start with

$$
S[\Phi, h]=\int d^{4} x\left[\partial \Phi^{*} \partial \Phi+\sum_{s} h_{s} J_{s}(\Phi)\right]+S[h], \quad S[h]=\kappa \sum_{s} \int h_{s} P_{s} \partial^{2 s} h_{s}+O\left(h^{3}\right)
$$


Then four-scalar tree-level scattering amplitude is found to be [2]

$$
A_{s}^{(\mathrm{t})}(\mathrm{s}, \mathrm{t}, \mathrm{u})=P_{s}\left(\frac{\mathrm{s}-\mathrm{u}}{\mathrm{s}+\mathrm{u}}\right)
$$

where $\mathrm{s}, \mathrm{t}, \mathrm{u}$ are Mandelstam variables: $\mathrm{s}+\mathrm{t}+\mathrm{u}=0$ and $P_{s}(x)$ is the Legendre polynomial (which is replaced by the Gegenbauer polynomial $C_{s}^{\left(\frac{d-3}{2}\right)}$ in $d$ dimensions). The amplitude is manifestly scaleinvariant. Summed over spins the t-channel amplitude is

$$
A^{(\mathrm{t})}(\mathrm{s}, \mathrm{t}, \mathrm{u})=\kappa^{-1} F_{4}\left(-\frac{\mathrm{s}-\mathrm{u}}{\mathrm{s}+\mathrm{u}}\right), \quad F_{4}(z)=\sum_{s=0}^{\infty}\left(s+\alpha_{d}\right) P_{s}(z), \quad \alpha_{d} \equiv \frac{d-3}{2}=\frac{1}{2}
$$

For generic $z$ the sum over spins diverges and requires a regularization. The same regularization as in (15) gives a delta-function: $F_{4}^{\text {reg }}(z)=\delta(z-1)$. Then the total amplitude for $\Phi \Phi \rightarrow \Phi \Phi$ scattering given by the sum of $t$-channel plus $u$-channel contributions) is

$$
A_{\Phi \Phi \rightarrow \Phi \Phi}=\kappa^{-1}\left[\delta\left(\frac{\mathrm{s}}{\mathrm{t}}\right)+\delta\left(\frac{\mathrm{s}}{\mathrm{u}}\right) .\right]
$$

It actually vanishes for physical momenta: in c.o.m. frame $\vec{p}_{1}+\vec{p}_{2}=0=\vec{p}_{3}+\vec{p}_{4}$ where in terms of the scattering angle $\frac{\mathrm{s}}{\mathrm{t}}=-\left(\sin ^{2} \frac{\theta}{2}\right)^{-1}, \quad \frac{\mathrm{s}}{\mathrm{u}}=-\left(\cos ^{2} \frac{\theta}{2}\right)^{-1}$ the arguments of delta-functions never vanish for real $\theta$, i.e. $A_{\Phi \Phi \rightarrow \Phi \Phi}=0$. Similarly,

$$
A_{\Phi \Phi^{*} \rightarrow \Phi \Phi^{*}}=\frac{\kappa^{-1}}{2}\left[\delta\left(\frac{\mathrm{u}}{\mathrm{t}}\right)+\delta\left(\frac{\mathrm{u}}{\mathrm{s}}\right)\right]=\frac{\kappa^{-1}}{2}\left[\delta\left(\cot ^{2} \frac{\theta}{2}\right)-\delta\left(\cos ^{2} \frac{\theta}{2}\right)\right]
$$

so here the t-channel and s-channel contributions cancel each other and again $A_{\Phi \Phi^{*} \rightarrow \Phi \Phi^{*}}=0$. Thus while the individual spin $s$ exchange contributions are nontrivial the total amplitude vanishes in particular summation prescription.

The reason is in the large underlying global HS symmetry that constrains the S-matrix (like in the case of hidden conserved charges in $2 \mathrm{~d}$ theories). The global part of the CHS symmetry contains the conformal generators plus other higher spin generators, e.g., "hyper-translations" $\delta \Phi=\varepsilon^{\mu_{1} \ldots \mu_{r}} \partial_{\mu_{1}} \ldots \partial_{\mu_{r}} \Phi$, For example, this fixes the $A_{\Phi \Phi \rightarrow \Phi \Phi}$ amplitude to be $A_{\Phi \Phi \rightarrow \Phi \Phi}(\mathrm{s}, \mathrm{t}, \mathrm{u})=$ $k_{1}(\mathrm{t}, \mathrm{u}) \delta(\mathrm{s})+k_{2}(\mathrm{~s}, \mathrm{u}) \delta(\mathrm{t})+k_{3}(\mathrm{t}, \mathrm{s}) \delta(\mathrm{u})$ In addition, the invariance under dilatations $p \rightarrow \lambda p$ $A_{\Phi \Phi \rightarrow \Phi \Phi}\left(\lambda^{2} \mathrm{~s}, \lambda^{2} \mathrm{t}, \lambda^{2} \mathrm{u}\right)=A_{\Phi \Phi \rightarrow \Phi \Phi}(\mathrm{s}, \mathrm{t}, \mathrm{u})$ then implies that the only solution consistent with crossing and scaling symmetry is

$$
A_{\Phi \Phi \rightarrow \Phi \Phi}(\mathrm{s}, \mathrm{t}, \mathrm{u})=0 \text {. }
$$

We conclude that the regularization in which the tree-level scalar amplitude vanishes is thus consistent with the CHS symmetry. This conclusion generalizes also to scattering amplitudes involving CHS fields on external lines [11].

\section{Scalar scattering in massless HS theory in flat space}

Massless 2-derivative higher spins define a unitary free theory $\left(\int d^{4} x \partial \phi_{s} \partial \phi_{s}, \delta \phi_{s}=\partial \epsilon_{s-1}\right)$ but it is an open question if there is a consistent interacting theory also in flat space. Such theory may be of interest if there is a flat space limit of the consistent MHS theory in AdS (given by Vasiliev's equations); it might be related to a tensionless limit of string theory, if such limit can be properly defined in flat space. It is not clear (in contrast to CHS case) which could be the underlying HS symmetry but one expects again an infinite tower of HS conserved charges. These should then constrain the S-matrix. To explore such theory one may use the known cubic couplings to compute some simple scattering amplitudes. 
Despite various $s>2$ "no-go theorems" prohibiting, in particular, minimal interactions (i.e. higher spin long-range forces) one may look for a consistent theory that contains (i) infinite tower of all spins $s=0,1,2,3, \ldots, \infty$; (ii) higher derivative (non-minimal) cubic interactions $\partial^{n} \phi_{s_{1}} \phi_{s_{2}} \phi_{s_{3}}, \quad s_{2}+s_{3}-$ $s_{1} \leq n \leq s_{2}+s_{3}+s_{1} \quad\left(s_{1} \leq s_{2} \leq s_{3}\right)$ (see a review in [1]). Using Noether procedure one may then deform the transformations $\delta \phi_{s}=\partial \epsilon_{s-1}+\ldots$, and attempt to determine the 4-point and higher interaction vertices. This should fix 3-point coupling constants like was done in the l.c. gauge in [9] $g_{s_{1} s_{2} s_{3}}=g \frac{\ell^{s_{1}+s_{2}+s_{3}-1}}{\left(s_{1}+s_{2}+s_{3}-1\right) !}$ The resulting theory should have two parameters: $g=$ dimensionless and $\ell=$ length

$$
S=\frac{1}{g^{2}} \int d^{4} x\left[\sum_{s} \partial \phi_{s} \partial \phi_{s}+\sum \ell^{n-1} \partial^{n} \phi_{s_{1}} \phi_{s_{2}} \phi_{s_{3}}+\sum \ell^{k-2} \partial^{k} \phi^{4}+\ldots\right]
$$

where $\phi^{3}$ terms contain two covariant structures $\partial^{s_{1}+s_{2}+s_{3}}$ and $\partial^{s_{2}+s_{3}-s_{1}}$. Whether there exists a consistent and local $\phi^{4}$ vertex remains an open question. The theory is in any case effectively non-local: the number of derivatives grows with $s$ and with number of fields $n$ in $\phi^{n}$ vertex. It may still have hidden simplicity under a particular summation over spins prescription.

It is useful to describe symmetric higher spin tensors by $\phi_{s}(x, u)=\phi^{a_{1} \ldots a_{s}}(x) u_{a_{1}} \ldots u_{a_{s}}$ where $u^{a}$ is a constant vector. Then the free Fronsdal action is

$$
\begin{aligned}
& S^{(2)}\left[\phi_{s}\right]=\int d^{d} x\left[\phi_{s}\left(x, \partial_{u}\right) K \phi_{s}(x, u)\right]_{u=0}, \\
& K=\left(1-\frac{1}{4} u^{2} \partial_{u}^{2}\right)\left[\partial_{x}^{2}-\left(u \cdot \partial_{x}\right)\left(\partial_{x} \cdot \partial_{u}\right)-\frac{1}{2}\left(u \cdot \partial_{x}\right) \partial_{u}^{2}\right]
\end{aligned}
$$

The off-shell field $\phi_{s}$ double-traceless $\left(\partial_{u}^{2}\right)^{2} \phi_{s}(x, u)=0$ and linearized gauge transformations are $\delta_{s}^{(0)} \phi_{s}(x, u)=\left(u \cdot \partial_{x}\right) \varepsilon_{s-1}(x, u)$. In de Donder gauge $\partial^{a_{1}} \phi_{a_{1} \ldots a_{s}}+\ldots=0$ the equations of motion become $\square \phi_{s}(x, u)=0$.

To discuss scattering of spin 0 particles: we need cubic interaction vertex with $\left(s_{1}=0, s_{2}, s_{3}\right)$ which reads $\left(\partial_{x_{i j}} \equiv \partial_{x_{i}}-\partial_{x_{j}}\right)$

$$
S^{(3)}\left[\phi_{0}, \phi_{s_{2}}, \phi_{s_{3}}\right]=g_{0 s_{2} s_{3}} \int d^{d} x\left[\left(\partial_{u_{2}} \cdot \partial_{x_{31}}\right)^{s_{2}}\left(\partial_{u_{3}} \cdot \partial_{x_{12}}\right)^{s_{3}} \phi_{0}\left(x_{1}\right) \phi_{s_{2}}\left(x_{2}, u_{2}\right) \phi_{s_{3}}\left(x_{3}, u_{3}\right)\right]_{u_{i}=0}
$$

The spin $s$ propagator is in $d=4$

$$
\begin{aligned}
& \mathcal{D}_{s}\left(u, u^{\prime} ; p\right)=-\frac{i}{p^{2}} \mathcal{P}_{s}\left(u, u^{\prime}\right), \quad \mathcal{P}_{s}\left(u, u^{\prime}\right)=\frac{1}{(s !)^{2}}\left(\sqrt{u^{2} u^{\prime 2}}\right)^{s} T_{s}\left(\frac{u \cdot u^{\prime}}{\sqrt{u^{2} u^{\prime 2}}}\right), \\
& T_{s}(z) \equiv \frac{s}{2} \sum_{k=0}^{[s / 2]} \frac{(-1)^{k}(s-k-1) !}{k !(s-2 k) !}(2 z)^{s-2 k}=\frac{1}{2}\left[\left(z+\sqrt{z^{2}-1}\right)^{s}+\left(z-\sqrt{z^{2}-1}\right)^{s}\right]
\end{aligned}
$$

where $T_{s}$ is the Chebyshev polynomial of first kind. Following [10] we may then compute the scattering of spin 0 fields via echange of the tower of massless HS fields with the above cubic interactions and making a conjecture about possible quartic coupling. One finds in s-channel [1]

$$
\begin{aligned}
& \mathcal{A}_{\text {exch }}(\mathrm{s}, \mathrm{t}, \mathrm{u})=\sum_{s=0,2,4, \ldots}^{\infty} \mathcal{A}_{\text {exch }}^{s}(\mathrm{~s}, \mathrm{t}), \quad \mathcal{A}_{\text {exch }}^{s}(\mathrm{~s}, \mathrm{t}, \mathrm{u})=-\frac{i g_{00 s}^{2}}{\mathrm{~s}}(\mathrm{t}+\mathrm{u})^{s} T_{s}\left(\frac{\mathrm{t}-\mathrm{u}}{\mathrm{t}+\mathrm{u}}\right) \\
& \mathcal{A}_{\text {exch }}(\mathrm{s}, \mathrm{t}, \mathrm{u})=-\frac{i}{\mathrm{~s}}[F(\sqrt{\mathrm{s}+\mathrm{t}}+\sqrt{\mathrm{t}})+F(\sqrt{\mathrm{s}+\mathrm{t}}-\sqrt{\mathrm{t}})] \\
& F(z) \equiv \sum_{s=0,2,4, \ldots}^{\infty} g_{00 s}^{2}\left(\frac{z^{2}}{4}\right)^{s}=\frac{1}{8} g^{2}(\ell z)^{2}\left[I_{0}(\ell z)-J_{0}(\ell z)\right]
\end{aligned}
$$


with the total result being

$$
\hat{\mathcal{A}}_{\text {exch }}(\mathrm{s}, \mathrm{t}, \mathrm{u})=\mathcal{A}_{\text {exch }}(\mathrm{s}, \mathrm{t}, \mathrm{u})+\mathcal{A}_{\text {exch }}(\mathrm{t}, \mathrm{s}, \mathrm{u})+\mathcal{A}_{\text {exch }}(\mathrm{u}, \mathrm{t}, \mathrm{s}) .
$$

The sum over spins here is convergent giving Bessel functions with non-trivial dependence on the Mandelstam variables and the scale $\ell$. In the Regge limit $\mathrm{t} \rightarrow \infty$, $\mathrm{s}=$ fixed

$$
\hat{\mathcal{A}}_{\text {exch }}(\mathrm{s}, \mathrm{t}, \mathrm{u}) \sim-\frac{i g^{2}}{\mathrm{~s}} \ell^{2} \mathrm{t} I_{0}(\ell \sqrt{8 \mathrm{t}}) \sim-\frac{i g^{2}}{\mathrm{~s}}\left(\ell^{2} \mathrm{t}\right)^{3 / 4} e^{\ell \sqrt{8 \mathrm{t}}}
$$

while in the fixed angle UV limit when $\mathrm{s}, \mathrm{t}, \mathrm{u} \rightarrow \infty, \frac{\mathrm{t}}{\mathrm{s}}=-\sin ^{2} \frac{\theta}{2}, \frac{\mathrm{u}}{\mathrm{s}}=-\cos ^{2} \frac{\theta}{2}$ we get

$$
\hat{\mathcal{A}}_{\text {exch }}(\mathrm{s}, \mathrm{t}, \mathrm{u}) \sim i g^{2}|\mathrm{~s}|^{3 / 4} e^{\ell \sqrt{|\mathrm{s}|} f(\theta)} \rightarrow \infty, \quad f(\theta)>0
$$

This amplitude has exponential UV growth. This may be compared to soft UV limit of the ShapiroVirasoro amplitude in string theory:

$$
\begin{aligned}
& A_{4}=g^{2} \frac{\Gamma\left(-1-\frac{1}{4} \alpha^{\prime} \mathrm{s}\right) \Gamma\left(-1-\frac{1}{4} \alpha^{\prime} \mathrm{s}\right) \Gamma\left(-1-\frac{1}{4} \alpha^{\prime} \mathrm{s}\right)}{\Gamma\left(2+\frac{1}{4} \alpha^{\prime} \mathrm{s}\right) \Gamma\left(2+\frac{1}{4} \alpha^{\prime} \mathrm{s}\right) \Gamma\left(2+\frac{1}{4} \alpha^{\prime} \mathrm{s}\right)} \\
& A_{4} \rightarrow g^{2}|\mathrm{~s}|^{-6}(\sin \theta)^{-6} e^{-\alpha^{\prime}|\mathrm{s}| h(\theta)} \rightarrow 0, \quad h(\theta)=-\frac{1}{4}\left(\sin ^{2} \frac{\theta}{2} \log \sin ^{2} \frac{\theta}{2}-\cos ^{2} \frac{\theta}{2} \log \cos ^{2} \frac{\theta}{2}\right)>0
\end{aligned}
$$

To get the full 4-point amplitude we need to add the contribution of 0-0-0-0 vertex. It is expected to be effectively "non-local" with infinite series in $\partial^{n}$; this may "soften" the UV behaviour of the exchange contribution. One may try to guess the 4-scalar vertex in flat-space HS action from its form in AdS action reconstructed using AdS/CFT in [12]. This leads to

$$
S^{(4)}\left[\phi_{0}\right]=g^{2} \int d^{4} x\left[\sum_{s=0}^{\infty} f_{2 s}\left(\Delta_{x_{34}}\right)\left(\partial_{x_{12}} \cdot \partial_{x_{34}}\right)^{2 s} \phi_{0}\left(x_{1}\right) \phi_{0}\left(x_{2}\right) \phi_{0}\left(x_{3}\right) \phi_{0}\left(x_{4}\right)\right]_{x_{i}=x}
$$

where $\Delta_{x_{34}} \equiv\left(\partial_{x_{3}}+\partial_{x_{4}}\right)^{2}, \quad \partial_{x_{12}} \equiv \partial_{x_{1}}-\partial_{x_{2}}$ and $f_{2 s}(z)$ is an infinite series in $z$, regular at $z=0$ :

$$
z \rightarrow \infty: \quad f_{2 s}(z) \rightarrow c_{2 s} \frac{\ell^{4 s-2}}{z}, \quad c_{2 s}=\frac{1}{[(2 s-1) !]^{2}}
$$

Then the asymptotic contribution to the 4-scalar amplitude is

$$
\sum_{s=0}^{\infty} f_{2 s}(\mathrm{~s})(\mathrm{t}-\mathrm{u})^{2 s}=\frac{2 \mathrm{t}+\mathrm{s}}{2 \mathrm{~s}}\left[I_{0}(2 \ell \sqrt{2 \mathrm{t}+\mathrm{s}})-J_{0}(2 \ell \sqrt{2 \mathrm{t}+\mathrm{s}})\right]
$$

This is similar to the above exchange contribution so some cancellation between the two remains a possibility. Constraints of gauge invariance on other amplitudes with HS particles on external lines suggest that quartic vertices should be non-local [13]. Assuming the existence of a consistent gaugeinvariant action for massless HS theory in flat space it may then turn out that the resulting S-matrix will be nearly trivial due to the cancellation between the exchange and 4-vertex contributions.

To conclude, we are beginning to learn how to do quantum computations in theories with infinite number of massless higher spin fields. This brings up the importance of proper definition of quantum theory (sum over spins, UV regularization) consistent with symmetries of the theory. The remarkable large symmetry of higher spin theories implies various simplifications (1-loop $Z=1$, zero effective number of d.o.f.) and simplification of S-matrix (at least in conformal HS theory).

This work was supported by the ERC Advanced grant No.290456 and by the Russian Science Foundation grant 14-42-00047 associated with Lebedev Institute. 


\section{References}

[1] D. Ponomarev and A. A. Tseytlin, "On quantum corrections in higher-spin theory in flat space," JHEP 1605, 184 (2016) [arXiv:1603.06273].

[2] E. Joung, S. Nakach and A. A. Tseytlin, "Scalar scattering via conformal higher spin exchange," JHEP 1602, 125 (2016) [arXiv:1512.08896].

[3] M. Beccaria and A. A. Tseytlin, "On higher spin partition functions," J. Phys. A 48 (2015) no.27, 275401 [arXiv:1503.08143].

[4] M. Beccaria and A. A. Tseytlin, "Higher spins in $\mathrm{AdS}_{5}$ at one loop: vacuum energy, boundary conformal anomalies and AdS/CFT," JHEP 1411, 114 (2014) [arXiv:1410.3273].

[5] M. Beccaria, X. Bekaert and A. A. Tseytlin, "Partition function of free conformal higher spin theory," JHEP 1408, 113 (2014) [arXiv:1406.3542].

[6] S. Giombi, I. R. Klebanov and A. A. Tseytlin, "Partition functions and Casimir energies in higher spin $\mathrm{AdS}_{d+1} / C F T_{d}$," Phys. Rev. D 90, no. 2, 024048 (2014) [arXiv:1402.5396].

[7] A. A. Tseytlin, Nucl. Phys. B 877, 598 (2013) [arXiv:1309.0785].

[8] S. Giombi, I. R. Klebanov and B. R. Safdi, "Higher Spin $\mathrm{AdS}_{d+1} / \mathrm{CFT}_{d}$ at One Loop," Phys. Rev. D 89, no. 8, 084004 (2014) [arXiv:1401.0825].

[9] R. R. Metsaev, "Poincare invariant dynamics of massless higher spins: Fourth order analysis on mass shell," Mod. Phys. Lett. A 6, 359 (1991).

[10] X. Bekaert, E. Joung and J. Mourad, “On higher spin interactions with matter," JHEP 0905, 126 (2009) [arXiv:0903.3338].

[11] M. Beccaria, S. Nakach and A. A. Tseytlin, "On triviality of S-matrix in conformal higher spin theory," arXiv:1607.06379 [hep-th].

[12] X. Bekaert, J. Erdmenger, D. Ponomarev and C. Sleight, "Quartic AdS Interactions in HigherSpin Gravity from Conformal Field Theory,” JHEP 1511, 149 (2015) [arXiv:1508.04292].

[13] M. Taronna, "Higher-Spin Interactions: four-point functions and beyond," JHEP 1204, 029 (2012) [arXiv:1107.5843]. 